\title{
Use of Individual Flight Corridors to Avoid Vortex Wakes
}

\author{
Vernon J. Rossow* \\ NASA Ames Research Center, Moffett Field, California 94035-1000
}

\begin{abstract}
Vortex wakes of aircraft pose a hazard to following aircraft until the energetic parts of their flowfields have decayed to a harmless level. It is suggested here that in-trail spacings between aircraft can be significantly and safely reduced by designing an individual, vortex-free flight corridor for each aircraft. Because each aircraft will then have its own flight corridor, which is free of vortex wakes while in use by the assigned aircraft, the time intervals between aircraft operations can be safely reduced to the order of seconds. The productivity of airports can then be substantially increased. How large the offset distances between operational corridors need to be to have them vortex free, and how airports need to be changed to accommodate an individual flight-corridor process for landing and takeoff operations, are explored. Estimates are then made of the productivity of an individual flight-corridor system as a function of the in-trail time interval between operations for various values of wake decay time, runway width, and the velocity of a sidewind. The results confirm the need for short time intervals between aircraft operations if smaller offset distances and increased productivity are to be achieved.
\end{abstract}

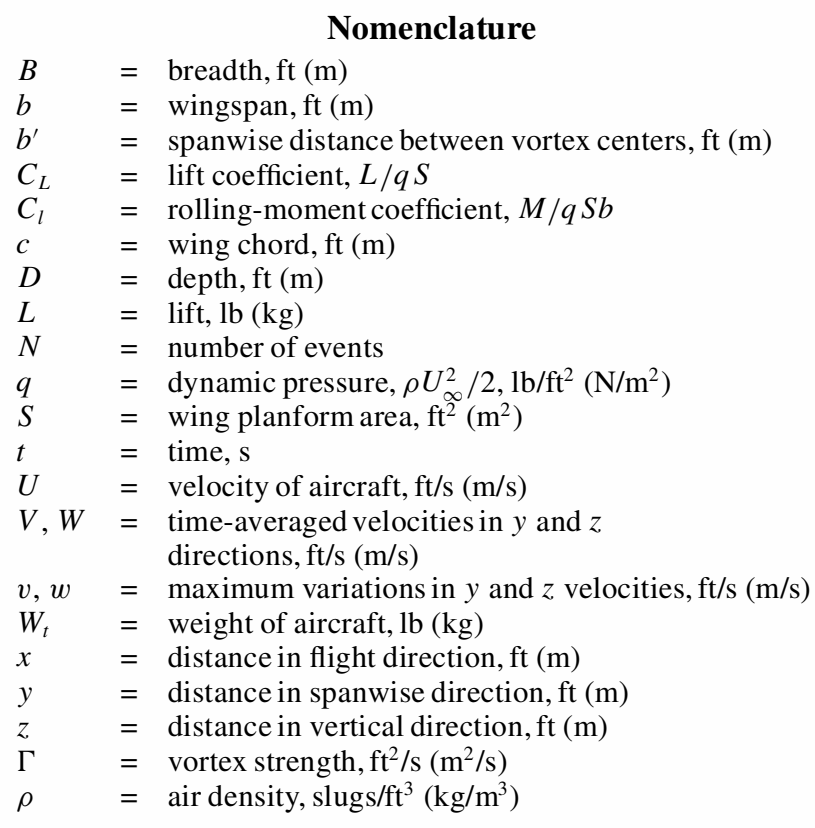

Subscripts

$\begin{array}{ll}\text { corr } & =\text { flight corridor } \\ \text { cycl } & =\text { sequence cycle } \\ \mathrm{dk} & =\text { wake decay } \\ \text { dlay } & =\text { cycle delay } \\ \text { eff } & =\text { effective } \\ f & =\text { following aircraft } \\ g & =\text { wake-generating aircraft } \\ \text { hz } & =\text { hazardous region of wake } \\ \text { offst } & =\text { offset distance } \\ \text { ops } & =\text { aircraft operations }\end{array}$

Received 20 December 2001; presented as Paper 2002-4874 at the AIAA Atmospheric Flight Mechanics Conference, Monterey, CA, 5-8 August 2002; revision received 23 October 2002; accepted for publication 25 October 2002. This material is declared a work of the U.S. Government and is not subject to copyright protection in the United States. Copies of this paper may be made for personal or internal use, on condition that the copier pay the $\$ 10.00$ per-copy fee to the Copyright Clearance Center, Inc., 222 Rosewood Drive, Danvers, MA 01923; include the code 0021-8669/03 $\$ 10.00$ in correspondence with the CCC.

* Senior Staff Scientist, Aerospace Operations Modeling Office, Mail Stop N210-10. Associate Fellow AIAA.

$$
\begin{array}{ll}
\text { prod } & =\text { productivity } \\
\text { slf } & =\text { self-induced } \\
\text { stn } & =\text { station } \\
\text { wnd } & =\text { wind } \\
0 & =\text { centerline } \\
\infty & =\text { freesteam condition }
\end{array}
$$

\section{Introduction}

$\mathbf{T}$ HE rolling-moment hazard posed by lift-generated vortex wakes during landing and takeoff operations at airports is a serious deterrent to capacity and safety. At present, hazardous regions of vortex wakes are avoided by maintaining enough in-trail separation distances, or times, between aircraft operations so that encounters do not occur. ${ }^{1-3}$ Safe in-trail separation distances were first determined during the 1960 s by means of Federal Aviation Administration (FAA) and NASA flight tests and by consideration of practices in use at airports at that time. Measurements made in the 1970s indicate that the time intervals now set between aircraft operations are long enough that the vortices have either moved out of the flight corridor to be used, or have decayed to a harmless level. ${ }^{2-7}$ Although the in-trail separation guidelines are effective, they limit the number of aircraft that can use a given airspace and runway per unit of time, and thereby the capacity of airports. If the present operational procedures are used, it is estimated that removal of the wake-vortex hazard as a limitation on spacing between aircraft would increase the capacity of airports by up to $10-20 \%$, depending on the mix of aircraft in the traffic flow and on the design of the airport. ${ }^{3}$ Even though such a capacity increase is not large, alternatives to the FAA in-trail spacing guidelines are being sought, because a number of airports in the United States are currently operating at or near capacity during certain times of the day. ${ }^{3,5}$

Beginning in the late 1960s, substantial research efforts were expended during a prolonged attempt to remove or reduce the effect of the hazard posed by vortex wakes on airport capacity. ${ }^{1-7}$ The research was either directed at the development of more efficient and effective avoidance methods, or at the modification of the aerodynamics of the wake-generating aircraft so that vortex wakes would not be hazardous to aircraft following at times as short as $1 \mathrm{~min}$. A 1-min separation time was chosen because, at that time, other factors at airports required that aircraft operations be separated by $1 \mathrm{~min}$ or more. Work in both of the foregoing research areas was usually restricted to finding solutions that could be implemented at airports or onto aircraft with relatively small changes from current practice. ${ }^{7}$ Even though the research efforts were broadly based and extensive, neither an avoidance method, nor an aerodynamic alleviation mechanism was found that both adequately reduced the hazard 
posed by vortex wakes and that also did not significantly penalize the wake-generating aircraft.

Since the beginning of the FAA/NASA research program on wake vortices in the late 1960 s, the need for a reduction in the FAA spacing guidelines during approach to and departure from airports has become more urgent because the volume of traffic has continued to increase. Along with a need for increased capacity at airports, it has also become more difficult to obtain public acceptance for new airports in the areas where they are most needed. Therefore, if the air transportation business is to satisfy the increasing demand for services, some way must be found to better utilize existing airport surface areas so that increasing traffic volumes can be accommodated. One constraint that should probably be retained in the development of new concepts is that the overall surface area of airports probably cannot be increased.

In the concept studied, each takeoff or landing operation is assigned a separate flight corridor and runway surface. Each flight corridor and runway combination (or aircraft conduit), is designed so that an encounter with the vortex wake of a preceding aircraft is avoided to a high degree of certainty. Based on available information on the motion and spread of the hazardous region associated with vortex wakes of aircraft, the sequential flight-corridor/runway conduits for aircraft are made vortex free by offsetting them by enough lateral distance so that the vortex wake of a leading aircraft does not contaminate the trajectory of any following aircraft. The concept studied is, therefore, labeled an individual flight-corridor concept because each aircraft must have its own flight corridor, which is designed specifically to avoid the vortex wakes of preceding aircraft. If found to be practical, the time intervals between aircraft landings and/or takeoffs can be reduced to a level that is based on factors other than the wake-vortex hazard.

It is apparent that the individual flight-corridor concept being studied is similar in some ways to existing flight corridor and runways systems at airports. ${ }^{8,9}$ The most obvious similarity is the use of parallel runways. The individual flight-corridor concept differs from current parallel-runway systems in that the number of runways used, and their lateral spacings, are aggressively controlled and arranged to optimize safely the number of aircraft operations per hour that can be accommodated by use of the available airport surface area. To achieve such a goal, runways are spaced laterally by minimum amounts based on the guidance capabilities available for aircraft, wind conditions, etc., rather than on large, fixed spacings designed to satisfy all possibilities. ${ }^{10}$ The reason for minimum lateral spacings is to utilize airport surface area efficiently by use of arrangements and procedures that safely enable the largest number of aircraft operations per unit of time, for example, before the air and runway spaces available at an airport need to be recycled for the next sequence of aircraft operations. The minimum required spacings are achieved in this study by aggressive use of all available information on aircraft guidance and wind conditions to predict more accurately the time-dependent locations of the aircraft involved and the vortex wakes that they shed. ${ }^{10-12}$ Also, implementation of the concept will require the use of air traffic management studies that improve the safety of operations with tools that prevent one aircraft from blundering into or interfering with operations on another closely spaced parallel runway. ${ }^{13-16}$ Therefore, the present study considers the individual flight-corridorconcept an extension of previous studies on closely spaced parallel-runway systems. The paper describes the individual flight-corridor concept, the tools needed for implementation, and some estimates of the airport productivity that such a process might provide.

\section{Individual Flight-Corridor Concept}

Becausedifferentmethodscan be used to generateoffset distances between flight corridors, the trajectories used for landing and taking off at airports is divided into two regions (Fig. 1). One part is labeled an outer corridor region because it is that part of a flight corridor where the altitude above ground level is high enough that glide-slope and slue-angle variations can be used to generate across flight-path distances between aircraft. Slue-angle variations have been used for some time under certain circumstances to enhance safety of flight.
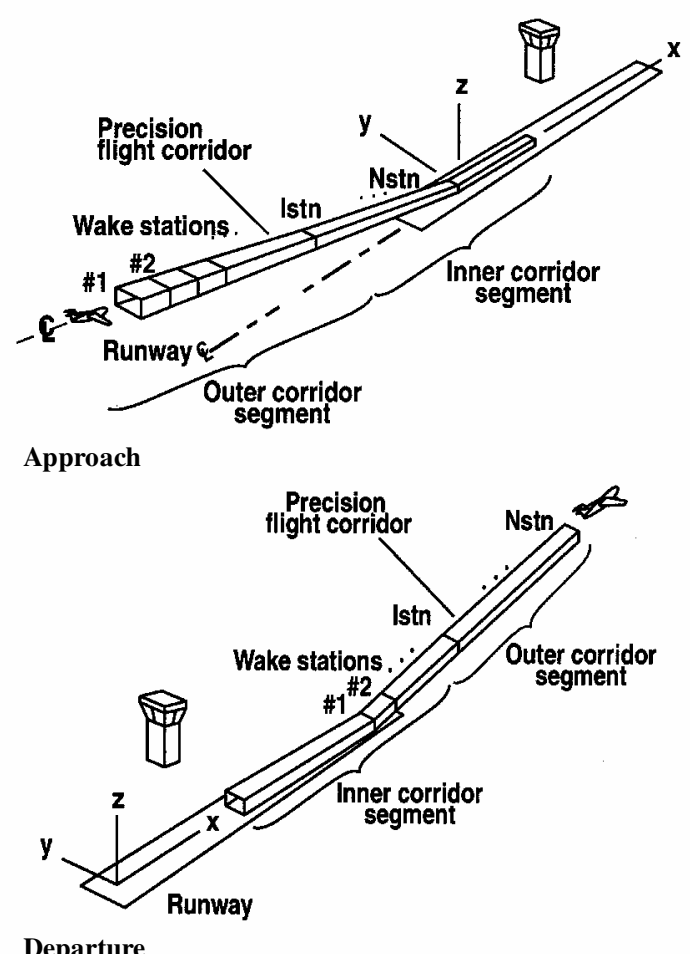

Departure

Fig. 1 Stations along precision flight corridor used to monitor beginning locations of vortex wakes; wake stations not shown. ${ }^{10}$

One example is the procedure used by the military to enhance the safety of flight during minimum-interval takeoffs. ${ }^{13}$ The process (called fan headings) requires that, just after becoming airborne, the lead aircraft turns to assume a heading downwind of the runway centerline. All following aircraft also turn just after liftoff to fly along headings that increase in the upwind direction by increments of 5-10 deg. As a result, after liftoff, all of the aircraft fan out in different directions.

Although the military version calls for heading changes in the horizontal plane only, the concept can be applied to directional changes in both the lateral (slue) and vertical (glide-slope) directions. ${ }^{10}$ The individual flight-corridor concept utilizes incremental changes in both angulardirectionsto enhance the safety of the process. Thereby, as the distance between aircraft flight paths is increased, the airspace being used can immediately be recycled because the use of heading changes in both the horizontal and vertical directions is much less sensitive to sidewinds than the use of only one of the parameters.

The second part, or inner region of the flight corridors, is where the approach or departure flight corridors are at an altitude so low that the proximity of the ground plane prohibits vertical offsets between flight corridors. Slue-angle variations are usually also inhibited along the inner segments of flight corridors because they are near touchdown or liftoff where the flight paths of aircraft need to be aligned with the centerline of their assigned runway path. Possible solutions for each of these two categories of flight-corridor regions or segments are discussed in the next two sections.

\section{Outer Part of Flight Corridors}

If airport capacity is to be significantly increased, it is not sufficient to just have more accurate information on the parameters that govern the transport and spread of vortex wakes. ${ }^{10}$ On single runways, it is the rapid spread and persistence of the hazardous region of vortex wakes that prevents the safe use of short time intervals between aircraft operations. Because some vertical and lateral airspace is available along the outer part of flight corridors for both landing and takeoff, it is suggested ${ }^{10}$ that the flight paths of a sequence of aircraft each have their own flight corridor and that the corridors be placed off to the side of and above or below other corridors by use of a sequence of slue and glide-slope angles (Fig. 2). It was found that 


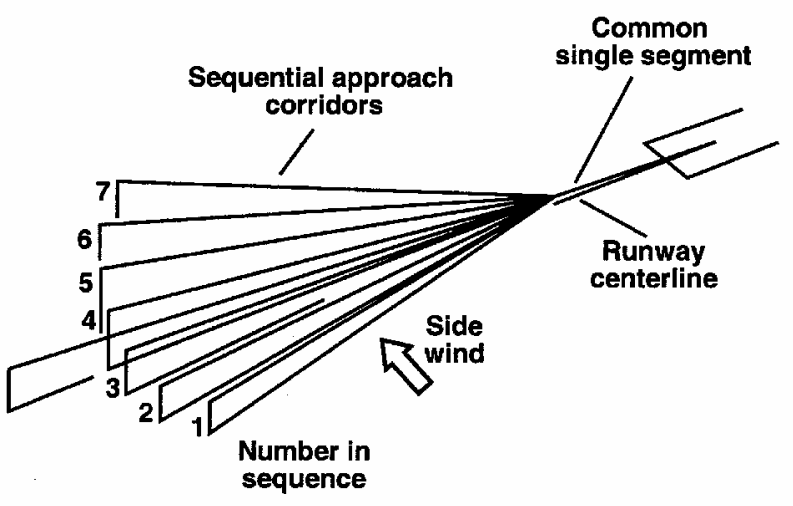

Fig. 2 Centerlines for flight corridors that use both glide-slope and slue-angle variations on outer segments of sequential two-segment approach flight corridors to avoid vortex wakes. ${ }^{10}$

aircraft would then be able to avoid with certainty the vortex wakes of preceding aircraft along the outer segments of the flight corridors. To reduce the clutter in Fig. 2, only the centerlines of the flight corridors, and not their boundaries, are shown. Note that the process avoids vortex encounters by generating across flight-path separation distances between corridors, rather than by increasing in-trail distances between aircraft. Adequate avoidance distances are achieved if the angular increments between successive approach corridors are equal to or greater than $0.5 \mathrm{deg}$ in glide slope, and $3 \mathrm{deg}$ in slue angle. ${ }^{10}$ The maximum slue angle is limited to $\pm 9 \mathrm{deg}$, so that aircraft can easily execute a turn from an incoming slued corridor to align themselves with the common segment and runway centerline. When the entire set of flight corridors has been used, the airspace must be recycled. One set of flight corridors (for example, for landing or for takeoff) is referred to as one cycle of flight corridors for a given sequence of aircraft operations.

The separation of flight corridors shown for the sequencein Fig. 2 is also effective when a sidewind is blowing in the direction of increasing slue angle. ${ }^{10}$ It was found that, along the outer segments of flight corridors, a sequence of corridors could then be recycled immediately after each foregoing cycle had been completed. Encounters with vortex wakes are avoided because the wind convects the wakes under flight corridors that are about to be used. However, if the sidewind blows in the direction opposite to the increasing slue angle, the vortex wakes of one cycle are blown onto the flight corridors to be used in the next cycle, which requires a time delay between cycles. Because sidewinds are not a problem when they are in the direction of increasing slue angle, the sequence of flight corridors can usually be set up so that slue angle always increases in the same direction as the sidewind. This option is available because, even though glide slope must increase whether slue-angle increases in the port or starboard direction, the slue angle can proceed either to port or to starboard. If this is done, glide-slope/slue-angle sequences are robust and wake-encounter probabilities are negligible everywhere except near the junction of the corridors and along the common flight-corridor segment just before touchdown. It is assumed, therefore, that the solution presented in Fig. 2 is adequate for the outer segments of flight corridors, and only the inner regions of flight corridors need to be treated.

\section{Inner Part of Flight Corridors}

Because, the use of rotations between flight corridors in the vertical (glide-slope) or lateral (slue) directions, illustrated in Fig. 2, can not be used while aircraft are near the ground, or near the end of a runway, some other way must be found to reduce the wakeencounter probability to zero along those parts of the trajectories. Near the ground, lateral offsets are the only means available for generating space between the inner parts of flight corridors, as illustrated in Fig. 3. However, lateral offsets must be applied along the entire length of flight corridors, including the outer parts of the flight corridors. In this way, separation distances between the inner regions of flight corridors are established by lateral offsets, and

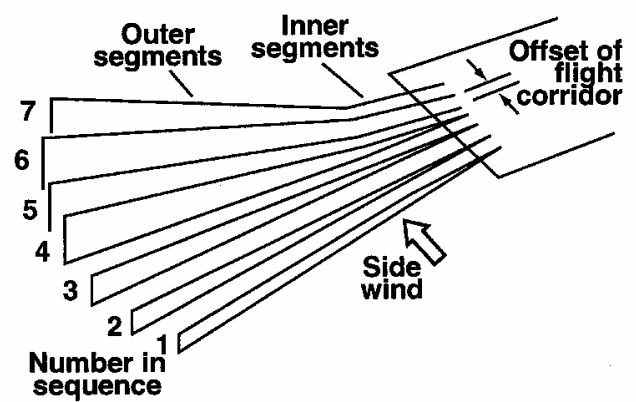

Fig. 3 Lateral offsets applied to entire length of flight corridors.

those brought about by glide-slope and slue-angle contributions are enhanced. As a consequence, safety of flight is established along the inner corridor regions and enhanced along outer segments. The magnitude of the offsets is governed by the characteristics of the wake-generating aircraft and of the atmospheric flowfield along the inner segment.

The process used to compute the amount of the lateral or horizontal offset is determined theoretically as the amount necessary to ensure that the entire length of an approach or departure corridor is free of vortex wakes while in use by a following aircraft. Various parameters are considered in the present work to determine which configurations and procedures provide the greatest efficiency with adequate safety. Application of the same concept to takeoff operations is accomplished by simply reversing the direction of motion of aircraft that use the flight corridors in Fig. 3. On departure, the maximum magnitude of slue and climbout angles can probably be increased over those used for approach.

\section{Equipment Required}

\section{Instrumentation}

Because the concept to be explored is based on having an individual flight corridor for each aircraft operation at an airport, the location and boundaries of the flight corridors must be preciselycontroled if the concept is to work most efficiently. Precise control of the location of aircraft and wake edges requires instrumentation with the capability to guide aircraft accurately along flight paths that are arbitrary within wide limits and within flight corridors that are small in cross section. ${ }^{10}$ Equipment with the required precision-guidance capability is being developed and is planned for implementation in the not too distant future. One set of equipment is based on the global positioning satellite(GPS) system as enhanced by some form of either the wide area augmentation system ${ }^{17-20}$ (WAAS), the local area augmentation system (LAAS), or by pseudosatellites. ${ }^{21-23}$ Although these systems are being developed primarily for airtraffic management purposes and zero-visibility landing capabilities, the equipment should also be able to provide the guidance accuracy needed for the implementation of an individual flight-corridor system.

\section{Runway Surface Areas}

Offsets along runways were dismissed early in the study because an examination of data on existing airports shows that today's runway surfaces are not sufficiently long ${ }^{8,9}$ to support an individual flight-corridor concept if vertical offsets (or offsets along runways) are used. For example, runways are usually long enough to fulfill the requirements of the aircraft expected at the airport, with very little extra available. Any extra lengths now present serve as a safety factor that can not be compromised. For example, runway lengths at major airports are about $5000 \mathrm{ft}$ long for general aviation and small commuter-type aircraft. Runway lengths are generally around $10,000 \mathrm{ft}$ long for transport-type aircraft. Some airports have runways of around 13,000 ft or more for military, larger transport, or high-altitude aircraft operations. ${ }^{9}$ It is concluded that not much, if any, runway length is available for extensive Offsets along runways. In addition, very little airport space is available for large extensions of runway length because space within the airport perimeter must be 


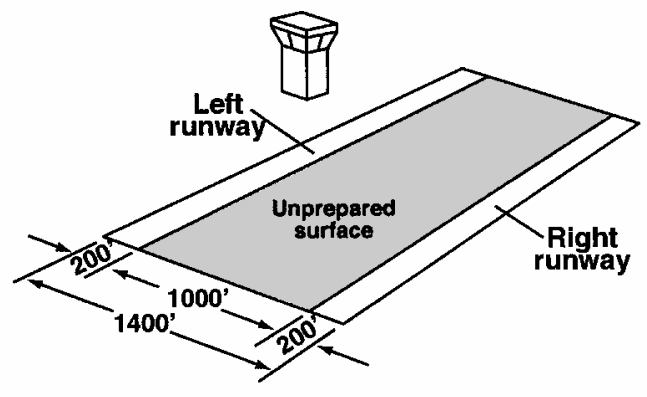

a) Two parallel runways

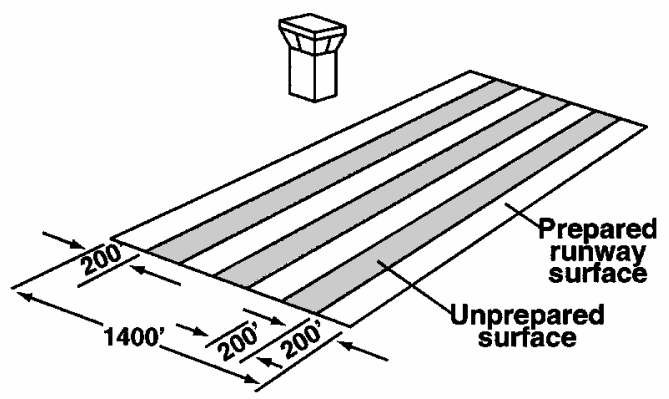

b) Two runways inserted to obtain four runways

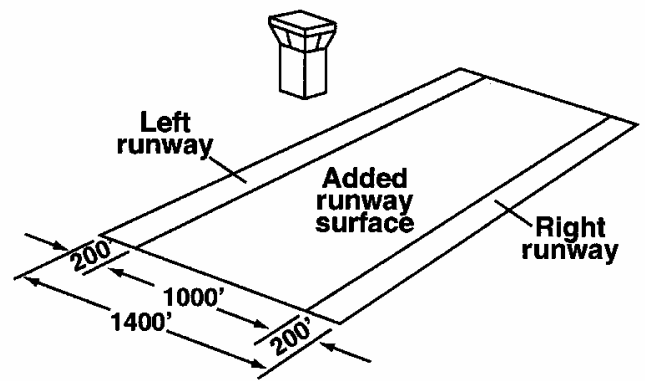

c) Entire surface prepared for arbitrary offsets

Fig. 4 Development of area between parallel runways to produce extra runway paths.

allowed for overruns and for low-altitude approach and departure flight paths. Therefore, it is probably not possible to extend runway lengths beyond those that currently exist at most airports and, therefore, offsets along runways will not be considered..$^{10}$

Offsets across runways do, however, appear to be feasible. Data on airport runways indicate that several possibilities exist. A single runway by itself is able to accommodate only one flight corridor because most runways are usually only about $200 \mathrm{ft}$ wide. ${ }^{8,9}$ Any new or revised runway surfaces must, therefore, be developed at the sides of, or between, existing runways, to achieve the needed surface widths. Based on typical configurations for parallel runways at major airports, it appears that a combination of the width of the two parallel runways and their separation distance will yield, for example, $200+200+1000 \mathrm{ft}$, or approximately $1400 \mathrm{ft}$ for the width of an enhanced runway surface that could be used for the present study (Fig. 4a). The 1400-ft width can be utilized by either placing additionalrunways between the existing ones (Fig. 4b), or by paving the entire area between parallel runways so that a continuous surface width would become available (Fig. 4c).

If discrete parallel runways (Fig. 4b) are placed between the two existing ones, the flight-corridor and runway offsets would become fixed at the largest required value, making the possible runway offsets less flexible. Such a runway pattern would expedite the removal of snow, ice, and water, but would limit the possibilities available for compact flight paths for short separation times and some wind conditions. The most flexible arrangement appears to be achieved by having the entire width of the available surface prepared for runway use (Fig. 4c). Enlargement of available runway width in this way may be considered as a first step toward a configuration where airport surfaces are completely paved to expedite aircraft operations.
Because some airports may be able to include taxiways for runway width, the variations possible from one airport to the next may be quite different. A runway surface $1400 \mathrm{ft}$ wide will, therefore, be used in the examples to be studied in the sections to follow.

\section{Design of Flight Corridor}

The flight corridor boundaries to be used for a sequence of aircraft are set up as described earlier (Fig. 1). The design of the flight corridors begins with a specification as to the location of the centerline of each flight corridor. The sides, top, and bottom of the flight corridors are next specified at regular intervals along the centerline by locating the upper, lower, port, and starboard sides of the corridor relative to the centerline of the corridor. In the present study, the cross-sectional shape of the flight corridor is always square, but the size of the cross section is variable.

To keep track of all possible locations of vortex wakes, "wake stations" are establishedat the same stations along the flight corridor. The wake-station boundaries are used as a computational tool to keep track of all possible locations for the wake-hazardous region shed by each aircraft. ${ }^{10}$ To make sure that all parts of the hazardous region are inside the boundaries of a wake station, the boundaries are moved with time in response to parameters that govern the motion and spreading of vortex wakes. The initial cross-sectional size of a wake station is made just large enough to include all possible beginning locations for the hazardous region of the vortex wake shed by each aircraft. Thereafter, the boundaries of each wake station are moved with time in response to local winds and an estimated spreading rate of the hazardous region. ${ }^{10}$ In this way, it is certain that the entire hazardous region of the vortex wake shed by each aircraft is contained within the wake-station boundaries. As a consequence, the analysis not only assigns each aircraft its own flight corridor, but each is also assigned its own set of wake-station boundaries whose interiors must be avoided by following aircraft.

The closed-form equations to be used here for the movement of wake-station boundaries are simplified versions of a numerical method used previously to carry out estimates of wake-encounter probability. ${ }^{10}$ The equations can be simplified because they are used only to estimate the lateral offset distances that are necessary to avoid the wake-hazardous regions shed by preceding aircraft.

\section{Computation of Lateral Offset Distance}

The parameters that determine the location of vortex wakes as a function of time, and the distance that a flight corridor must be shifted laterally to avoid the hazardousregion behind a preceding aircraft, include the following five factor ${ }^{10}: 1$ ) location of flight path of wake-generating aircraft, 2) size and location of its wake-hazardous region, 3) self-induced descent velocity of wake, 4) wind velocity in vicinity of wake, and 5) location of flight path of following aircraft.

In the simplified analysis, only factors $1,2,4$, and 5 need to be considered because the vertical distances brought about by the selfinduced downward velocity of the wake can be ignored. If safety of flight for each following aircraft is to be assured, the offset distance must be large enough that no part of the wake-hazardous region of any preceding aircraft overlaps with the flight corridor being used by the following aircraft, that is, every flight corridor must be vortex free.

A plan view of how lateral offset distance is related to other parameters in the airport environment is presented in Fig. 5. The centerlines and boundaries of the flight corridors for a wake-generating and a following aircraft indicate how they are separated from each other by an offset distance. Also shown are the lateral motion of the wake due to the sidewind and the spreading rate of its hazardous region. The size of the wake-hazardous region increases with time due to atmospheric affects, and self-induced instabilities of the vortex pair. The increase in cross-sectional size is represented by the same functionaldependence derived from data taken on the condensation trails behind aircraft at cruise altitudes. ${ }^{24}$ The initial size of the wake-hazardous region is based on the rolling-moment hazard posed by the rolled up configuration of the wake vortices. When the following aircraft is the same size as the wake-generating aircraft, the breadth of the wake-hazardous region begins at about 2.5 spans 


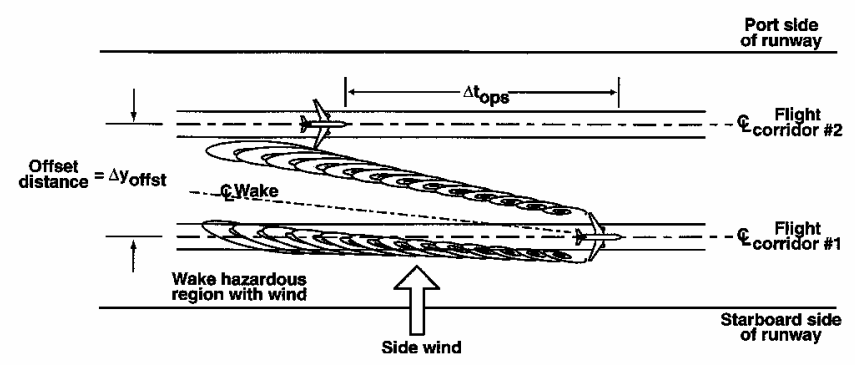

Fig. 5 Flight corridors and vortex wake used to compute offset distance between centerlines of a wake-generating and following aircraft.

(associated offset equals 1.25 spans). Thereafter, the hazardous region is approximated by a function that takes into account the size of the wake as it grows due to instabilities and diffusion given by

$$
D_{\mathrm{hz}} \approx B_{\mathrm{hz}}(t) \approx b_{g} C_{\mathrm{hz}} \sqrt{\Delta t}
$$

where,

$$
C_{\mathrm{hz}} \approx 0.5
$$

Here, $t$ begins when the wake is generated and $\Delta t$ is the time interval between the arrival of the wake generator and following aircraft at the same in-trail station. The size of the wake-hazardous region remains constant until it exceeds the initial value of 2.5 spans, at which time the wake grows according to Eq. (1) (Refs. 10, 24). In other words, the wake remains constant until the spreading rate represented by Eq. (1) exceeds 2.5 spans. According to such a relationship, the crossover size of the depth of the hazardous region occurs when the time is $4 \mathrm{~s}$. Similarly, the breadth is predicted to exceed the beginning size at $16 \mathrm{~s}$ for small span ratios, and $25 \mathrm{~s}$ when the following aircraft is of the same or larger size. ${ }^{10}$ In the computations, all aircraft are assumed to be the same size and to have wing spans of $200 \mathrm{ft}$.

In Fig. 5, the yaw of the boundaries and centerline of the wake due to the sidewind are indicated by the asymmetry of the wakehazardous region relative to the centerline of the aircraft flight corridor. Based on Eq. (1), the lateral offset distance of the centerline of the flight corridor for a following aircraft from the centerline of the flight corridor of a preceding aircraft is equal to

$$
\Delta y_{\text {offst }} \geq B_{\mathrm{hz}}(t) / 2+B_{g \text { corr }} / 2+B_{f \text { corr }} / 2+\left|V_{\text {eff }}\right| \Delta t_{\text {ops }}
$$

In Eq. (2), $\Delta y_{\text {offst }}$ identifies the breadth of the wake-hazardous region, $B_{\mathrm{hz}}(t)$ the parameter, $B_{g \text { corr }}$ the breadth of the flight corridor being used by the wake-generatingaircraft, and $B_{f \text { corr }}$ the breadth of the flight corridor being used by the following aircraft. The breadth of the flight corridor for both the wake-generating and following aircraft are assumed to be the same and to be constant with time. The influence of a sidewind on offset distance is expressed by the last term in Eq. (2), $V_{\text {eff }}$, which is also assumed to be constant with time, and to represent the magnitude of that part of the sidewind that is effective in translating the hazardous region of a vortex wake laterally with time.

Because offset distances and productivity are based on time intervals between aircraft, increments in time are used instead of time from some beginning point. For this reason, the time intervals between operations are represented in Eq. (2) $\Delta t_{\mathrm{ops}}$. An allowance is not made for the self-induced downward velocity of the vortex pair, because it is directed downward and, therefore, only contributes to lateral offset when ground effect causes a sideways motion in the vortices, which is assumed to be included in the quantity $B_{\mathrm{hz}}(t)$. Note that Eq. (2) contains the parameters that are most significant in the determination of the offset distance. If a more complete and time-dependent simulation of the individual flight-corridor process was to be carried out, the complexity of the computation would increase substantially. ${ }^{10}$ If that were done, however, it is doubtful that the offset distances, productivity, and conclusion derived here would be greatly changed.

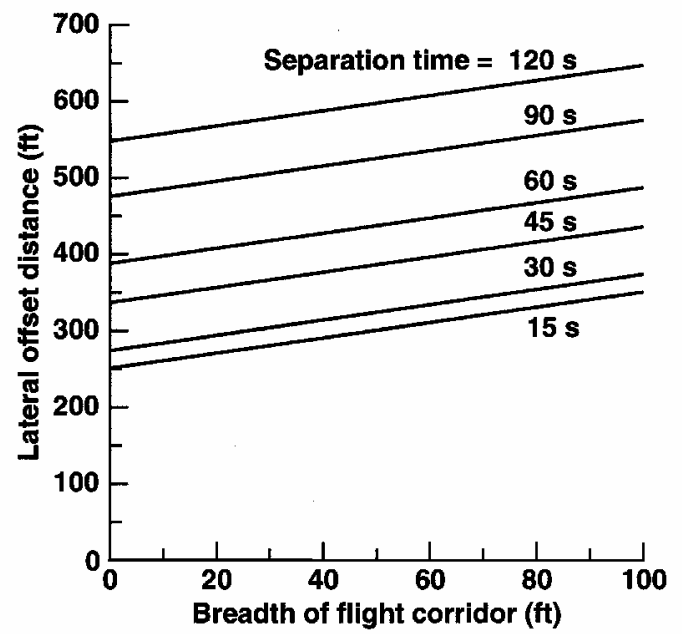

Fig. 6 Lateral offset distance as a function of breadth of the flight corridors for various separation times between aircraft operations: $b_{g}$ $=200 \mathrm{ft}$ and $V_{\text {eff }}=0$.

An illustration of how the various parameters in Eq. (2) affect offset distance is presented in Fig. 6 as a function of the breadth of the flight corridor. It is assumed that the sidewind is negligible, that is, $V_{\text {eff }}=0$. If the location of both the wake-generating and following aircraft can be controlled with precision, and the time interval between aircraft is a fraction of $1 \mathrm{~min}$, the offset distances become reasonable. Because the wingspan of the wake-generating aircraft used in the computation is $200 \mathrm{ft}$, and the following aircraft is assumed to have the same wingspan, the minimum offset distance is computed as $250 \mathrm{ft}$ for separation time intervals of $15 \mathrm{~s}$ or less. Therefore, if aircraft arrive or depart at closely spaced time intervals, the offset distances required are slightly larger than the wingspan of the wake-generating aircraft involved. Throughout the analysis, it was found that small in-trail separation times reduce offset distances and increase productivity because wake spreading and convection do not then have time to progress to where they require large offset distances.

\section{Productivity of Lateral Offsets}

\section{Equations to be Used}

As assumed in Fig. 6, the results to be presented assume that every aircraft involved in each example has a wingspan of $200 \mathrm{ft}$, and that they are each able to keep their centerlines within a flight corridor that has a $10 \times 10 \mathrm{ft}$ cross section. As stated earlier, the outer regions of flight corridors are assumed to be separated not only by lateral offsets, but also by slue and glide-slope variations, as illustrated in Figs. 2 and 3, so that those portions of the flight corridors are also free of vortex wakes. The analysis can then be restricted to the low-altitude portions of the operations being considered. Also, vertical distances are ignored to obtain a preliminary estimate of the productivity of various individual flight-corridor configurations and procedures. The information is presented not as a design study, but as exploratory considerations that might lead to a method for increasing the productivity of airports.

In the first set of examples, the runway breadth is assumed to be $1400 \mathrm{ft}$ (Fig. 4c), and the lateral offset distance is computed for each aircraft by the use of Eq. (2). The first aircraft is assigned to a flight corridor with its centerline located $100 \mathrm{ft}$ from the beginning edge of the runway surface, as if it were on a runway that is $200 \mathrm{ft}$ wide. The centerline for the flight corridor to be used by the second aircraft in the sequence is then located at a lateral distance of $100 \mathrm{ft}$ plus $\Delta y_{\text {offst }}$, from the same beginning side edge of the runway surface. The centerlines of each following aircraft in the sequence are likewise placed farther and farther from the beginning edge of the runway surface until lateral space is no longer available on the prepared surface of the runway.

If more aircraft are to be accommodated, the runway surface areas and their corresponding airspaces that were used before must 
be recycled. Before the next cycle begins, the vortex wakes of the preceding aircraft must either have decayed to a harmless level, or must have been blown out of the region to be used. The decay time for the vortex wakes is first assumed to be $3 \mathrm{~min}$, or $180 \mathrm{~s}$. A 3 -min wake decay time is about 1 min longer than current maximum separation times used at airports between in-trail aircraft and is, therefore, conservative. Each wake begins its decay process when it is deposited at a given wake station, and so the delay between cycles can be shortened by the time required for the preceding cycle of aircraft to be completed. The time to complete one cycle of aircraft operations is then given by

$$
\Delta t_{\mathrm{cycl}}=N_{\mathrm{ops}} \Delta t_{\mathrm{ops}}
$$

where $N_{\text {ops }}$ is the number of aircraft operations in one cycle of a sequence, and $\Delta t_{\mathrm{ops}}$ is the time interval between aircraft operations. The time-delay increment $\Delta t_{\text {dlay }}$ between cycles of operations is then given by

$$
\Delta t_{\mathrm{dlay}}=\Delta t_{\mathrm{dk}}-\Delta t_{\mathrm{cycl}}
$$

If the time delay is negative, it is set equal to zero. The productivity of the configuration being analyzed is then equal to the number of aircraft operations per cycle times the number of cycles per hour, as

$$
N_{\text {prod }}=3600 N_{\text {ops }} /\left(\Delta t_{\text {dlay }}+\Delta t_{\text {cycl }}\right)
$$

where the various time intervals are in seconds, and $N_{\text {prod }}$ is in aircraft operations per hour.

\section{No Sidewind}

Figure 7 presents the estimated productivity as a function of the time intervalbetween aircraftoperations, when no sidewind is blowing. As expected, when the surface area delegated to a runway is held constant, the productivity increases roughly as $1 / \Delta t_{\mathrm{ops}}$. The productivity is sensitive to the time required for the vortex wake to decay to a harmless level. As a reference curve, a dashed horizontal line is shown to indicate the level where 60 aircraft operations per hour, or one per minute, occurs

The dotted curve indicates the productivity for a single runway in the idealized case where the hazard posed by vortex wakes is not a problem. If such a situation were brought about by the invention of a highly effective wake-alleviation mechanism, in-trail spacings could be set to any length of time. The dotted curve then represents the maximum productivity that can be achieved with a single runway as a function of time interval between aircraft operations. The curves in Fig. 7 are shown down to a time interval between aircraft operations of $2 \mathrm{~s}$, because values down to zero are not meaningful in this analysis. In-trail spacings of $5 \mathrm{~s}$ or less could be termed almost simultaneous operations. The results emphasize the advantages of

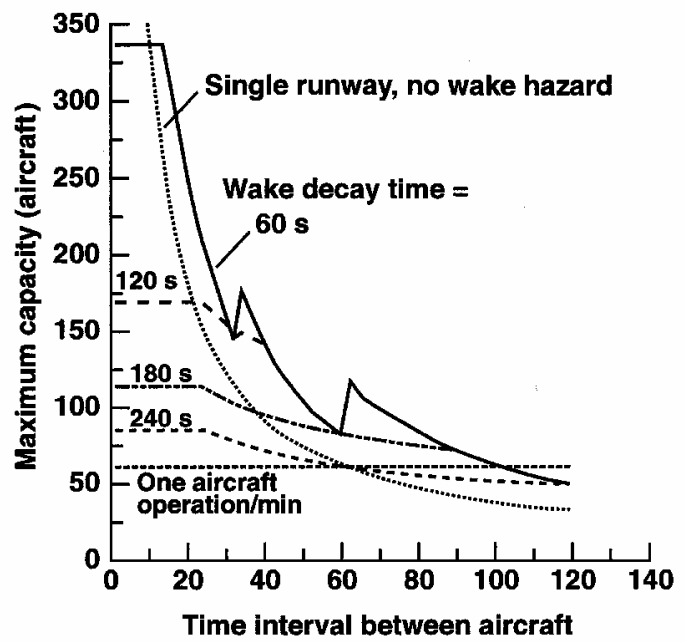

Fig. 7 Maximum productivity of runway design as a function of time interval between aircraft operations for several values of wake decay time: $b_{g}=200 \mathrm{ft}$, runway breadth $=1400 \mathrm{ft}$, and $V_{\text {eff }}=0$. small in-trail time intervals and show that they are essential for small offset distances and for high productivity.

Small separation times are probably the safest way to avoid vortex wakes because large uncertainties in wake location do not have time to develop. A lower limit on time intervals between aircraft will no doubt be placed on operations by the necessity to allow time for irregular acceleration and deceleration when on the ground and for turns onto and off of runways to and from loading areas. A lower limit based only on the vortex wake hazard is, however, not necessary if an individual flight-corridor process is implemented.

Note that the curve for a decay time of $60 \mathrm{~s}$ is above the dotted curve, thereby indicating a higher productivity than is possible with a single runway. The 60 -s curve exceeds the single runway curve because the procedures used for the individual flight-corridor system assumes that, if wake decay permits, the first aircraft operation of one cycle occurs at the same time as the last operation of the preceding cycle. Because aircraft are separated by the width of the runway minus $200 \mathrm{ft}$, and the wake is assumed to have decayed, such a procedure is safe and permissible. Other types of simultaneous operations were not considered. The three steps in the curve for a 60-s decay time occur because the number of aircraft operations per cycle is a discrete number. Not clearly indicated in Fig. 7 is that the curve for a wake decay time of $120 \mathrm{~s}$ joins the 60 -s curve and then follows it.

In summary, the results presented in Fig. 7 emphasize two important facts about airport capacity. First, small time intervals between aircraft operations expedite traffic volume because offset distances are small for short time intervals. Second, even though vortex wakes are avoided during each cycle by use of flight corridors, the efficiency of runway space is decreased by the time delay required between cycles to allow vortex wakes to decomposed to a harmless level.

\section{Effect of Runway Breadth on Productivity}

Because the breadth of the prepared runway surface area is not easily changed, and is a relatively fixed parameter for most airports, the productivity of various runway widths are compared in Fig. 8. Once again, a horizontal dashed line is used to indicate the productivity of 60 aircraft operations per hour. As in Fig. 7, the most important parameter is the time interval between aircraft operations. Surprisingly, as the time interval increases above about $40 \mathrm{~s}$, the productivity of all runway widths are about the same. However, at small values of time between operations, runway width is an importantfactor and should be utilized. Runway width appears to ensure that the time required for the aircraft operations in one cycle of use is long enough for the wakes to decay to a harmless level so that delay times between cycles are negligible. Possible simultaneous parallel operations over the wider runway surfaces were not considered because

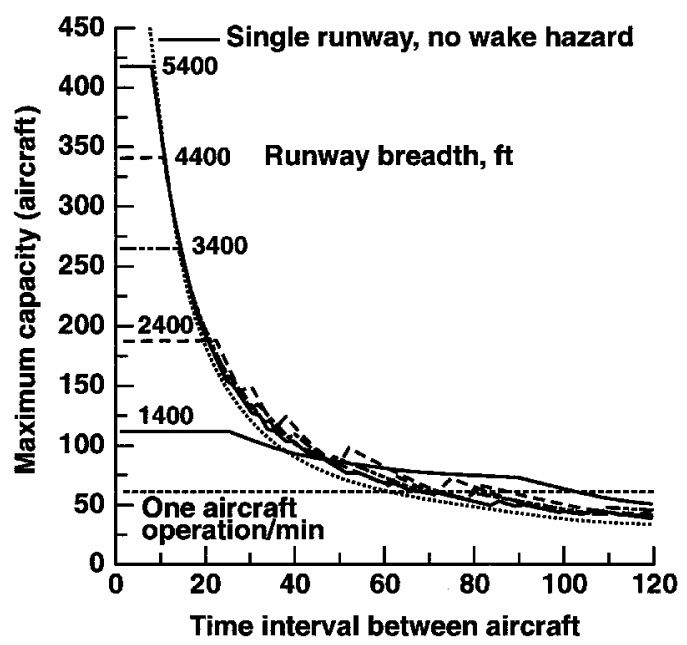

Fig. 8 Maximum productivity of runway design as a function of time interval between aircraft operations for several values of runway breadth: $b_{g}=200 \mathrm{ft}$, wake decay time $=180 \mathrm{~s}$, and $V_{\text {eff }}=0$. 


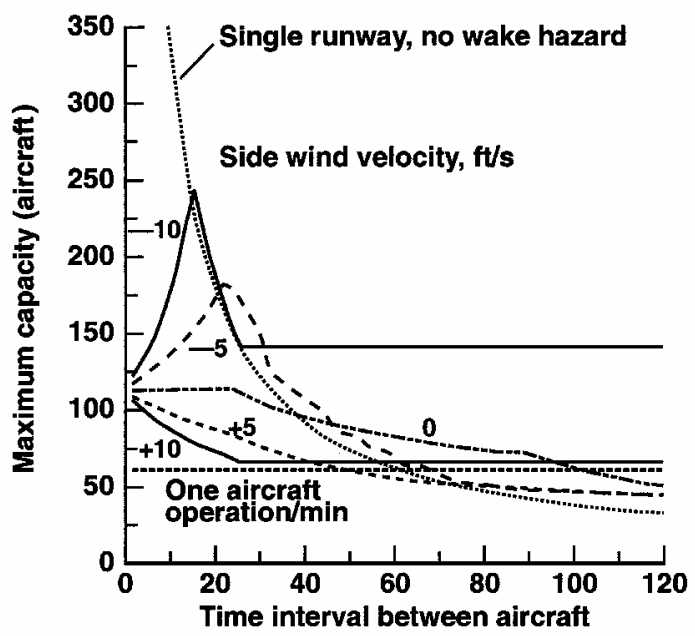

Fig. 9 Maximum productivity of runway design as a function of time interval between aircraft operations for several values of sidewind velocity: $b_{g}=200 \mathrm{ft}$, wake decay time $=180 \mathrm{~s}$, and runway breadth $=1400 \mathrm{ft}$.

the spread and drift of vortex wakes from one area of operation to another is not easy to treat with the simplified analysis used here.

\section{Effect of Sidewind on Productivity}

If a measurable sidewind is blowing, the offset distances must be adjusted accordingly. When the time intervals between aircraft operations are large (on the order of 1 min or more), even a small sidewind is capable of moving a vortex wake a considerable distance. However, at small time intervals between aircraft operations, the wind-driven displacements of wakes are correspondingly small and may not be of much consequence. If the sidewind is blowing strongly, the lateral convection of wakes is enough at moderate and long values of $\Delta t_{\mathrm{ops}}$ to generate vortex-freeregions that reduce offset distances to zero. When this occurs, the productivity curves become horizontal for sidewind velocities of -10 and $+10 \mathrm{ft} / \mathrm{s}$, as indicated in Fig. 9. At moderate sidewind velocities, the offset distances are increased enough so that the number of aircraft operations per cycle is small, so that recycle delay times significantly reduce productivity. In addition, when a sidewind is blowing in a direction opposite to the lateral offset distance, the time required for wakes to decay to a harmless level must be added to the cycle time because the wakes are convected into the beginning region of each cycle on the runway surface.

\section{Conclusions}

The study reported here on the use of individual flight corridors to guide aircraft along paths that avoid vortex wakes of preceding aircraft appears to have the capability to provide a significant increase in airport productivity. The process does require that instrumentation and procedures be developed and implemented for precise control of flight paths and for short time intervals between aircraft operations. From an aerodynamic point of view, the process studied appears to be effective and practical. The concept provides flexible scheduling so that time intervals between aircraft operations may vary from small values to values as large as desired. Note, however, that short time intervals between aircraft operations are essential for small offset distances between flight corridors and, consequently, for increased airport productivity. The concept suggested here appears to be able to shift the constraint on airport capacity from the hazard posed by lift-generated vortex wakes to other operational factors on the airport property.

\section{References}

${ }^{1}$ Gessow, A. (ed.), NASA Symposium on Wake Vortex Minimization, NASA SP-409, 1976.

${ }^{2}$ Hallock, J. N. (ed.), Proceedings of the Aircraft Wake Vortices
Conference-Vol. 1 and 2, U.S. Dept. of Transportation, Federal Aviation Administration, DOT/FAA/SD-92/1.1, DOT-VNTSC-FAA-92-7.1, Washington, DC, 1991.

${ }^{3}$ Hinton, D. A., "Aircraft Vortex Spacing System (AVOSS) Conceptual Design," NASA TM 110184, Aug. 1995.

${ }^{4}$ Wood, W. D. (ed.), FAA/NASA Proceedings Workshop on Wake Vortex Alleviation and Avoidance, Rept. FAA-RD-79-105, U.S. Dept. of Transportation, 1978

${ }^{5}$ Posluns, H. (ed.), Proceedings of the International Wake Vortex Meeting, 1997.

${ }^{6}$ Rossow, V. J., and Tinling, B. E., "Research on Aircraft/Vortex-Wake Interactions to Determine Acceptable Level of Wake Intensity," Journal of Aircraft, Vol. 25, No. 4, 1988, pp. 481-492.

${ }^{7}$ Rossow, V. J., "Lift-Generated Vortex Wakes of Subsonic Transport Aircraft," Progress in Aerospace Sciences, Vol. 35, No. 6, 1999, pp. 507660.

${ }^{8}$ Horonjeff, R., and McKelvey, F. X., Planning and Design of Airports, 4th ed., McGraw-Hill, New York, 1994, pp. 249-291.

9 "Low Altitude United States Airport Diagrams, Instrument Approach Procedures, Military Departure Procedures, Radar Instrument Approach Minimums," Department of Defense Fight Information Publication (Terminal), Vol. 2, National Imagery and Mapping Agency, NIMA Ref. No. NSN7641014005597, St. Louis, MO, 20 May 1999.

${ }^{10}$ Rossow, V. J., "Reduction of Uncertainties in Prediction of WakeVortex Locations," Journal of Aircraft, Vol. 39, No. 4, 2002, pp. 587596.

${ }^{11}$ Spitzer, E. A., Hallock, J. N., and Wood, W. D., "Status of the Vortex Advisory System," Proceedings of the Aircraft Wake Vortices Conference, edited by J. N. Hallock, Rept. FAA-RD-77-68, U.S. Dept. of Transportation, 1977, pp. 326-334.

${ }^{12}$ Hallock, J. N., Whitney, J. E., and Burnham, D. C., "Wind Criteria to Relieve Wake Vortex Effects on Departure," AIAA Paper 99-3420, JuneJuly, 1999.

${ }^{13}$ Gilbert, T., "Aerodynamic Influences During Minimum Interval Takeoffs and Aerial Refueling," Proceedings of the Aircraft Wake Vortices Conference-Vol. 1 and 2, edited by J. N. Hallock, U.S. Dept. of Transportation, Federal Aviation Administration, DOT/FAA/SD-92/1.1, DOT-VNTSCFAA-92-7.1, Washington, DC, 1991, pp. 12-1-12-10.

${ }^{14}$ Burnham, D. C., Hallock, J. N., and Greene, G. C., "Wake Turbulence Limits on Paired Approaches to Parallel Runways," Journal of Aircraft, Vol. 39, No. 4, 2002, pp. 630-637.

${ }^{15}$ Slattery, R. A., Lee, K. K., and Sanford, B. D., "Effects of ATC Automation on Precision Approaches to Closely Spaced Parallel Runways," 8th International Symposium on Aviation Psychology, AIAA Paper 95-3367, AIAA, Washington, DC, 1995, pp. 1769-1775.

${ }^{16}$ Koczo, S., "Coordinated Parallel Runway Approaches," NASA CR 201611, Rockwell International, Cedar Rapids, IA, Oct. 1996.

${ }^{17}$ Loh, R., Shively, C., and Swider, R., "Overview of theFAA's Differential GPS CAT III Technical Feasibility Demonstration Program," AIAA Guidance, Navigation, and Control Conference, AIAA Paper 93-3836, AIAA, Washington, DC, 1993.

18“Specification, Wide Area Augmentation System (WAAS)," U.S Dept. of Transportation, Federal Aviation Administration, FAA-E-2892, Washington, DC, May 1994.

${ }^{19}$ Paielli, R. A., McNally, B. D., Bach, R. E., Jr., and Warner, D. N., Jr., "Carrier Phase Differential GPS for Approach and Landing: Algorithms and Preliminary Results," Inst. of Navigation, 1993 International Technical Meeting (GPS-93), Salt Lake City, UT, Sept. 1993.

${ }^{20}$ Paielli, R. A., Bach, R. E., McNally, B. D., Jr., Simmons, R. C., Warner, D. N., Jr., Forsyth, T. J., Kanning, G., Ahtye, C. T., Kaufmann, D. N., and Walton, J. C., "Carrier Phase Differential GPS Integrated with an Inertial Navigation System: Flight Test Evaluation with Auto-Coupled Precision Landing Guidance," Inst. of Navigation, 1995 National Technical Meeting, Anaheim, CA, Jan. 1993.

${ }^{21}$ Pervan, B. S., "Navigation Integrity for Aircraft Precision Landing Using the Global Positioning System," Dept. of Aeronautics and Astronautics, SUDAAR Rept. 677, Stanford Univ., Stanford, CA, March 1996.

${ }^{22}$ Lawrence, D. G., "Aircraft Landing Using GPS: Development and Evaluation of a Real Time System for Kinematic Positioning Using the Global Positioning System," Dept. of Aeronautics and Astronautics, SUDAAR Rept. 690, Stanford Univ., Stanford, CA, Sept. 1996.

${ }^{23}$ Cobb, H. S., "GPS Pseudolites: Theory, Design, and Applications," Dept. of Aeronautics and Astronautics, SUDAAR Rept. 707, Stanford Univ., Stanford, CA, Sept. 1996.

${ }^{24}$ Rossow, V. J., and James, K. D., "Overview of Wake-Vortex Hazards During Cruise," Journal of Aircraft, Vol. 37, No. 6, 2000, pp. 960-975. 